\title{
HIGH RESOLUTION VLBI OBSERVATIONS OF THE NUCLEI OF BRIGHT GALAXIES AND QUASARS AT $327 \mathrm{MHz}$
}

\author{
S.Ananthakrishnan and V.K. Kulkarni \\ Radio Astronomy Centre(TIFR), P.O.Box 8, \\ Uthagamandalam 643001, India
}

\section{INTRODUCTION}

An intercontinental VLBI experiment at $327 \mathrm{MHz}$ involving the Ooty Radio Telescope and telescopes at Jodrell Bank, Westerbork, Torun and Crimea was carried out during December 8-12, 1983. The purpose of the experiment was to (i) establish the feasibility of performing MKII VLBI observations from India and (ii) to study the low frequency structure of nearby galaxies, quasars and some other well known radio sources at metre wavelengths. The sources observed were NGC 262, 315, 1052, 1068, 1265, 1275, 4151, 4486, 7674 and 3C 120; PKS 1055+018, 1148-001 and CTA 102; 3C 237 and SS 433. The bright NGC galaxies chosen were selected from an Interplanetary Scintillation survey of 150 nearby galaxies (Bagri and Ananthakrishnan 1983); they were found to have a flux density greater than about $0.2 \mathrm{Jy}$ in subarcsec components at $327 \mathrm{MHz}$ and have high frequency VLBI observations. The quasars chosen are amongst the most compact sources known at metrewavelengths. The other objects were the well known sources which have been studied extensively at higher frequencies.

\section{OBSERVATIONS AND ANALYSIS}

A total of 40 hours of simultaneous observations using the above stations were made. Since this was a pilot survey, each source was observed only for a few hours. The recording of the data was done at each of the stations using a MKII VLBI terminal with a bandwidth of $2.0 \mathrm{MHz}(326.0-328.0 \mathrm{MHz})$. The processing and analysis of the data were done at the MPI fur Radioastronomie, Bonn, FRG, using the MKII VLBI processor.

\section{RESULTS}

Table 1 gives the name of the source, correlated flux density for the shortest and longest baselines (corresponding to about $0.5 \times 10^{6}$ and $7 \times 10^{6} \lambda$ ) and for some of the sources, angular sizes derived from model fitting. It is seen from Table 1 that most of the nearby bright galaxies are found to be resolved on the longest baselines. Based on this pilot survey, another VLBI experiment is planned at $327.0 \mathrm{MHz}$ with a view to making detailed maps of some of the above sources for studying the low frequency structure. 


\section{Reference}

Bagri,D.S. and Ananthakrishnan,S., Proc. of IAU Symp.110 on 'VLBI and Compact Radio Sources', 261p.

Table 1

\begin{tabular}{llccc}
\hline Source Name & \multicolumn{2}{c}{$\begin{array}{l}\text { Correlated flux density in Jy } \\
\text { Largest } \\
\text { baseline }\end{array}$} & $\begin{array}{l}\text { Shortest } \\
\text { baseline }\end{array}$ & $\begin{array}{c}\text { Largest angular size } \\
\text { in mas }\end{array}$
\end{tabular}

$\begin{array}{cccc}\text { NGC } 262 & <0.1 & 0.4 & - \\ 315 & <0.1 & 0.3 & - \\ 1052 & <0.1 & 0.5 & - \\ 1068 & \sim 0.1 & 1.2 & - \\ 1265 & <0.1 & 0.15 & 30^{2} \\ 1275 & 2.0 & 7.5 & 400^{2} \\ 4151 & <0.1 & 0.25 & - \\ 4486 & <0.2 & 1.3 & - \\ 7674 & <0.1 & 0.8 & 160^{3} \\ 3 \text { C } 120 & 0.4 & 2.7 & 15^{4} \\ 1055+018 & 1.5 & 3.1 & 12^{4} \\ 1148-001 & 1.6 & 2.9 & 30^{5} \\ \text { CTA } 102 & 2.2 & 5.5 & 1200^{6} \\ 3 \text { C 237 } & <0.1 & 20.0 & 860^{4} \\ \text { SS } 433 & <0.1 & 0.5 & \end{array}$

\section{Notes}

1 Hybrid map being made.

2 Double; separation 400 mas; each component of size 100 mas; Ratio of Flux densities 2:1.

3 Double; separation 160 mas; component sizes 9 and 90 mas with flux density ratio $1: 5$.

4 Single component size.

5 Double; separation 30 mas; component sizes 12 and 18 mas with flux density ratio 3:2.

6 Double; separation 1200 mas; each component of size 250 mas; flux density ratio $1: 1$. 